\title{
Uniform semiclassical theory of avoided crossings
}

\author{
T. Uzera) \\ A. A. Noyes Laboratory of Chemical Physics, California Institute of Technology, Pasadena, California 91125 \\ D. W. Noid \\ Oak Ridge National Laboratory, Oak Ridge, Tennessee 37830
}

R. A. Marcus

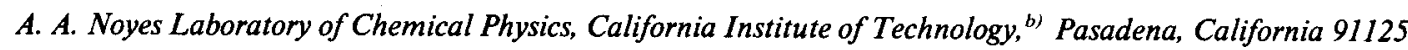

(Received 25 May 1983; accepted 23 July 1983)

\begin{abstract}
Avoided crossings influence spectra and intramolecular redistribution of energy. A semiclassical theory of these avoided crossings shows that when primitive semiclassical eigenvalues are plotted vs a parameter in the Hamiltonian they cross instead of avoiding each other. The trajectories for each are connected by a classically forbidden path. To obtain the avoided crossing behavior, a uniform semiclassical theory of avoided crossings is presented in this article for the case where that behavior is generated by a classical resonance. A low order perturbation theory expression is used as the basis for a functional form for the treatment. The parameters in the expression are evaluated from canonical invariants (phase integrals) obtained from classical trajectory data. The results are compared with quantum mechanical results for the splitting, and reasonable agreement is obtained. Other advantages of the uniform method are described.
\end{abstract}

\section{INTRODUCTION}

Potential energy curves (or surfaces) that approach each other closely in a narrow region without intersecting are a familiar feature in the description of collision processes within the Born-Oppenheimer approximation. The existence of such "avoided crossings" (or "anticrossings") for a certain parameter (e.g., the internuclear distance in atom-atom scattering) signals regions where couplings neglected in the Born-Oppenheimer approximation become appreciable.' The behavior of a variety of atomic and molecular processes, such as charge transfer, ${ }^{2}$ mutual neutralization, ${ }^{3}$ and predissociation ${ }^{4}$ are dominated by these regions.

Recently, avoided crossings have received additional attention in connection with the study of coupled anharmonic vibrations. ${ }^{5-14}$ In that context, avoided crossings occur in plots of eigenvalues of the relevant Hamiltonian vs a perturbation parameter coupling the vibrations. As noted elsewhere, there is a connection between these avoided crossings and the existence of an "isolated resonance" in the classical mechanics of anharmonic systems, in that an isolated resonance can, in quantum mechanics, produce an isolated avoided crossing. $5,7,11,14$ Whether it produces a large splitting or not depends on the "width" of the resonance zone, e.g., whether that classical width in action-variable space is large enough to contain the pair of quantum states, as we shall see. Isolated avoided crossings are of interest for several reasons: They produce local changes in the spectrum, extensive changes in the wave functions, and in the energy distribution among participating oscillators. When their widths overlap sufficiently, they can lead to the onset of a type of "chaos" in the wave functions and in the spectra,, 71,14 just as "overlapping" classical resonances are implicated in producing, in Chirikov theory, ${ }^{15}$ the onset of classical chaos. This distinction between overlapping and isolated avoided crossings (namely, that only the former has been related to chaos) is sometimes overlooked, for example. ${ }^{16}$

The study of classical chaos (associated with irregular spectra) and classical quasiperiodicity (associated with regular spectra) in anharmonically coupled oscillators has been of considerable interest in recent years (cf. Refs. 11,15, and 17-22 for reviews). The corresponding study in quantum mechanics of coupled oscillators, the sources of regular progressions in the spectrum, and of irregularities, of "regular" contour patterns of plots of wave functions vs highly "irregular" ones, is of similar interest. The present article is part of a series aimed at providing a semiclassical theory of "quasiperiodic" anharmonically coupled vibrations in molecules and in the present case, of their avoided crossings.

Recently, Noid et al. ${ }^{12}$ investigated quantal, classical, and semiclassical behavior of two anharmonically coupled oscillators at an isolated avoided crossing. In the quantum mechanics, the avoided crossing arose from a term in the Hamiltonian which produced a classical resonance. However, the "primitive semiclassical" eigenvalue plots, obtained by quantizing the action integrals of the classical trajectories, passed through the intersection instead of avoiding each other. The splitting is due, thereby, to a classically forbidden process. To obtain an "avoidance," a uniform semiclassical approximation, alluded to there, is desirable and is given in the present article.

The outline of the present article is as follows: to motivate a functional form chosen for the present uniform approximation we first derive in Sec. II a low-order classical perturbation expression for the energy that takes into account the classical internal resonance, Eq. (2.27). We then show in Sec. III how to evaluate the parameters in this equation using canonical invariants (phase integrals) obtained from classical trajectory data. The uniform semiclassical quantization of this classical Hamiltonian demonstrates 
how a classical resonance produces an avoided crossing. The expressions are given in Sec. IV. Remarks on the relation between widths of classical resonances and the splittings of avoided crossings are given in Sec. V. In Sec. VI numerical results are given on splittings, and primitive and uniform semiclassical eigenvalues are compared. A discussion and concluding remarks are given in Secs. VII and VIII, respectively.

\section{CONSTRUCTION OF THE RESONANCE HAMILTONIAN}

The semiclassical study of coupled oscillator systems has proceeded along two different lines. One of these is the numerical evaluation of phase integrals $\oint p d q$ using classical trajectories. ${ }^{11,23}$ The second consists of attempts to express the Hamiltonian in terms of good, or nearly so, action variables using perturbation or perturbation-iteration methods. ${ }^{11,18,24,25}$ An advantage of this second method is that the resulting analytic form may reveal information about the dependence of energy levels on the various parameters of the problem. (One disadvantage is that it diverges. ${ }^{26}$ ) In the present section, we use the perturbative method to obtain an analytical functional form [Eq. (2.27)], to treat the avoided crossing behavior.

To illustrate this procedure, we write the Hamiltonian of two coupled harmonic oscillators considered in Ref. 12 as

$$
H=H_{0}\left(I_{x}, I_{y}\right)+\epsilon V\left(I_{x}, I_{y}, \varphi_{x}, \varphi_{y}\right),
$$

where $\mathbf{I}=\left(I_{x}, I_{y}\right)$ are action-like variables, canonically conjugate to the angles $\varphi_{x}, \varphi_{y}$. These variables are related to the customary action-angle variables $\left(J_{i}, w_{i}\right) \quad(i=x, y)$ by $I_{i}=J_{i} / 2 \pi$ and $\varphi_{i}=2 \pi w_{i}$. The unperturbed Hamiltonian is

$$
H_{0}=\omega^{0} \cdot \mathbf{I}=\omega_{x}^{0} I_{x}+\omega_{y}^{0} I_{y},
$$

where $\omega^{0}=\left(\omega_{x}^{0}, \omega_{y}^{0}\right)$ are the unperturbed angular frequencies. $\epsilon V$ is a nonlinear coupling. Later, we shall designate these unperturbed $I$ 's and $\varphi$ 's appearing in Eqs. (2.1) and (2.2) by 0 superscripts. Progressively better action-angle variables (no superscript labels) can be constructed by a sequence of canonical transformations $s^{24,25,27}$ and the resulting Hamiltonian can be quantized by semiclassical quantization rules. The construction of successive canonical transformations becomes very inefficient, or indeed may fail, when the perturbed frequencies $\omega_{i}=\partial H / \partial I_{i}$ satisfy a "resonance condition"

$$
\sum_{i=x, y} m_{i} \omega_{i}(\mathbf{I})=0
$$

\footnotetext{
$H$ can be expressed in terms of the action-angle-like variables $\left(I_{i}, \varphi_{i}\right)$ of the unperturbed problem using the transforma-
} tion

$$
x=\left(2 I_{x} / \omega_{x}^{o}\right)^{1 / 2} \sin \varphi_{x}, \quad p_{x}=\left(2 I_{x} \omega_{x}^{0}\right)^{1 / 2} \cos \varphi_{x},
$$

and similarly for $y$. $H$ becomes

$$
\begin{aligned}
H= & 3 I_{x}+I_{y}-\frac{a}{4}\left[\left(2 I_{x} / 3\right)^{3 / 2}\left(-\sin 3 \varphi_{x}+3 \sin \varphi_{x}\right)+\left(2 I_{y}\right)^{3 / 2}\left(-\sin 3 \varphi_{y}+3 \sin \varphi_{y}\right)\right] \\
& -\frac{b}{\sqrt{3}}\left(I_{x} I_{y}^{3}\right)^{1 / 2} \sin \varphi_{x}\left(-\sin 3 \varphi_{y}+3 \sin \varphi_{y}\right)+\frac{\lambda}{3} I_{x} I_{y}\left(1-\cos 2 \varphi_{y}\right)\left(1-\cos 2 \varphi_{x}\right) .
\end{aligned}
$$

Near a resonance, the terms with $\left(l_{x}, l_{y}\right)$ that fulfill the resonance condition (2.3) have the slowest variation with time. Under such conditions, one may restrict attention to $H_{0}$ and to that part of $\epsilon V$ in Eq. (2.1) that depends on the angles in the combination

$$
\alpha=m_{x} \varphi_{x}+m_{y} \varphi_{y}
$$

with ( $\left.m_{x}, m_{y}\right)$ obtained from Eq. (2.3). The resulting Hamiltonian, known as the "resonance Hamiltonian," $H_{R},{ }^{15,20,29}$ is given explicitly as ${ }^{30}$

$$
H_{R}=\sum_{N=-\infty}^{N=\infty} H_{N}\left(I_{x}, I_{y}\right) \exp i N \alpha,
$$

where $H_{N}$ is the Fourier coefficient

$$
\begin{aligned}
H_{N}= & (2 \pi)^{-2} \int_{0}^{2 \pi} \int_{0}^{2 \pi} d \varphi_{x} d \varphi_{y} \\
& \times \exp (-i N \alpha) H\left(I_{x}, I_{y}, \varphi_{x}, \varphi_{y}\right)
\end{aligned}
$$

This method of secular perturbations was applied by Born in his studies in old quantum theory. ${ }^{27}$ In this procedure, one makes a transformation to "fast" and "slow" phases $(\alpha$ is the slow phase), and eliminates the fast phase by averaging over it. Consequently, the action conjugate to the fast phase becomes a constant of the motion.

We apply this method to the avoided crossing problem studied by Noid et al. ${ }^{12}$ They examined the energy levels of two coupled harmonic oscillators using the Hamiltonian

$$
\begin{aligned}
H= & \frac{1}{2}\left(p_{x}^{2}+p_{y}^{2}+\omega_{x}^{0^{2}} x^{2}+\omega_{y}^{0^{2}} y^{2}\right) \\
& -a\left(x^{3}+y^{3}\right)+\lambda x^{2} y^{2}-b x y^{3}
\end{aligned}
$$

with $\omega_{x}^{0}=3 \omega_{y}^{0}=3$. The energy levels were computed quantum mechanically and semiclassically as a function of $\lambda$ at fixed $a$ and $b$. Because of the zeroth order commensurability of the unperturbed frequencies $\omega_{x}^{0}$ and $\omega_{y}^{0}$, a nearly exact 3:1 occured for some $\left(I_{x}, I_{y}\right)$ interval at relatively small $\lambda$. We examine the energy levels of this Hamiltonian using its resonance Hamiltonian $H_{R}$.

with $\left\{m_{i}\right\}$ denoting integers. (The exact $\omega_{i}$ 's in a quasiperio-
dic motion depend on the action variables $\mathbf{I}$.) Small, or vanwill occur in the explicit construction and thus cause diffiory. To treat a Hamiltonian involving an isolated resonance ${ }^{29}$ it is convenient to use the double Fourier series of the 
The commensurability or "resonance condition"

$$
\omega_{x}=3 \omega_{y}
$$

for some $\lambda$ and $\mathbf{I}$ (the $\omega$ 's are the actual frequencies) indicates that one should make a canonical transformation ${ }^{31}$ to a set of slow and fast angles. We use the generating function

$$
F_{2}\left(\varphi_{x}, \varphi_{y} ; I_{\alpha}, I\right)=\left(\varphi_{x}-3 \varphi_{y}+\pi\right) I_{\alpha} / 2+I \varphi_{y}
$$

and find from

$$
\begin{aligned}
& \alpha=\partial F_{2} / \partial I_{\alpha}, I_{x}=\partial F_{2} / \partial \varphi_{x}, \text { etc. } \\
& 2 \alpha=\varphi_{x}-3 \varphi_{y}+\pi, \quad \theta=\varphi_{y}, \\
& I_{\alpha}=2 I_{x}, \quad I=3 I_{x}+I_{y} .
\end{aligned}
$$

The $\frac{1}{2}$ and the $\pi$ in Eq. (2.11) are introduced so as to obtain the $+\cos 2 \alpha$ in Eq. (2.27) later, without requiring a second transformation.

The Fourier components $H_{N}$ associated with the slow phase $\alpha$ can be found from Eq. (2.6) as

$$
\begin{aligned}
H_{N}= & (2 \pi)^{-2} \int_{0}^{2 \pi} \int_{0}^{2 \pi} d \varphi_{x} d \varphi_{y} H\left(I_{x}, I_{y}, \varphi_{x}, \varphi_{y}\right) \\
& \times \exp \left[-i N\left(\varphi_{x}-3 \varphi_{y}+\pi\right) / 2\right] .
\end{aligned}
$$

One obtains

$$
\begin{aligned}
H_{N}= & \left(3 I_{x}+I_{y}+\frac{\lambda}{3} I_{x} I_{y}\right) \delta_{N, 0} \\
& -\frac{b}{4 \sqrt{3}}\left(I_{x} I_{y}{ }^{3}\right)^{1 / 2}\left(\delta_{N,-2}+\delta_{N, 2}\right) .
\end{aligned}
$$

The resonance Hamiltonian is, from Eq. (2.7),

$$
\begin{aligned}
H_{R} & =\sum_{N=-\infty}^{N=\infty} H_{N} \exp i N \alpha \\
& =3 I_{x}+I_{y}+\frac{\lambda}{3} I_{x} I_{y}-\frac{b}{2 \sqrt{3}}\left(I_{x} I_{y}^{3}\right)^{1 / 2} \cos 2 \alpha .
\end{aligned}
$$

The resulting expression does not contain any terms due to the other perturbation $-a\left(x^{3}+y^{3}\right)$, and without them the avoided crossings would occur only at $\lambda=0$. The effect of that particular perturbation, calculated by second order canonical perturbation theory, ${ }^{27}$ is $E^{(2)}$ :

$$
E^{(2)}(\mathbf{I})=-\frac{15 a^{2}}{4}\left(\frac{I_{x}^{2}}{\omega_{x}^{0^{4}}}+\frac{I_{y}^{2}}{\omega_{y}^{0^{4}}}\right) .
$$

Thus, a resonance Hamiltonian that includes the effects of all three perturbations to their lowest orders is

$$
\begin{aligned}
H_{R}= & 3 I_{x}+I_{y}+\frac{\lambda}{3} I_{x} I_{y}-\frac{b}{2 \sqrt{3}}\left(I_{x} I_{y}^{3}\right)^{1 / 2} \\
& \times \cos 2 \alpha-\frac{15 a^{2}}{4}\left(\frac{I_{x}^{2}}{81}+I_{y}^{2}\right) .
\end{aligned}
$$

The above deviation of Eq. (2.17) is somewhat piecemeal but with some physical insight. A systematic but more cumbersome derivation of the same expression is obtained using Birkhoff-Gustavson perturbation theory. ${ }^{25,32}$ That formalism collects various perturbations in powers of coordinates and momenta rather than in powers of a single perturbation parameter and is therefore suited to our problem of multiple perturbations. It is given and applied in Appendix A.

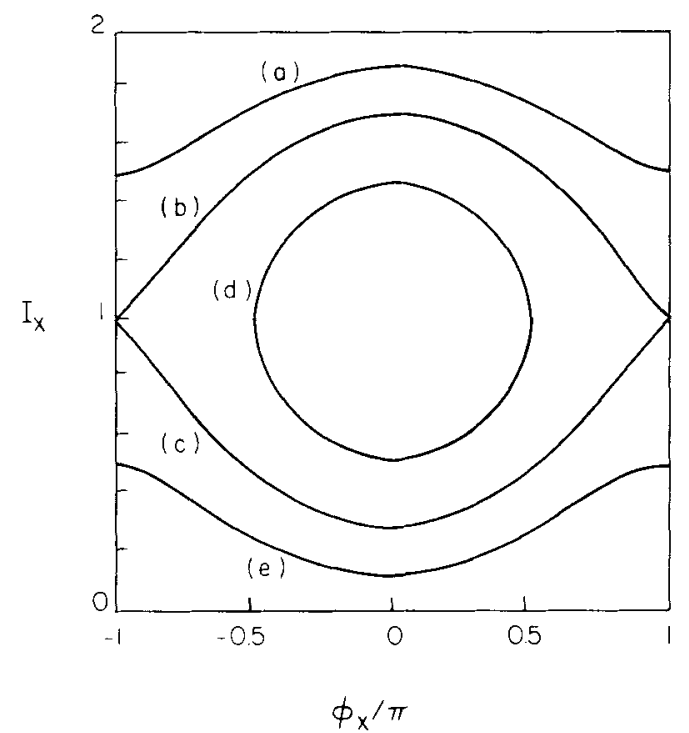

FIG. 1. Typical $I_{x}$ vs $\varphi_{x}$ plots. Curves (a) and (e) are rotational, (d) is librational, and (b) and (c) are the two branches of the separatrix.

One point is worth noting: The Birkhoff-Gustavson derivation of Eq. (2.17) reveals that the $I_{x}, I_{y}$ appearing there are no longer the original variables $I_{x}^{0}, I_{y}^{0}$ but are related to them by the successive canonical transformations. From Appendix A, Eq. (A18), we see that the first generating function of this succession of generating functions, relating old and new Cartesian variables, is

$F_{2}(\mathbf{q}, \mathbf{P})=\sum_{k=1}^{2} P_{k} q_{k}-\frac{a}{3} \sum_{k=1}^{2} \omega_{k}^{0^{-5 / 2}}\left(2 P_{k}^{3}+3 P_{k} q_{k}^{2}\right)$

which, to lowest order in $a$, gives the relation between the "old" and the "new" action-angle variables as

$$
I_{k}=I_{k}^{0}-\left(\frac{2}{\omega_{k}^{0}}\right)^{5 / 2} a\left(I_{k}^{0}\right)^{3 / 2} \sin ^{3} \varphi_{k}^{0},
$$

$\tan \varphi_{k}=\tan \varphi_{k}^{0}-\omega_{k}^{0-5 / 2}\left(2 I_{k}^{0}\right)^{1 / 2} a\left(2+\sin ^{2} \varphi_{k}^{0}\right) \sec \varphi_{k}^{0}$.

Consequently, $I_{x}^{0}$ vs $\varphi_{x}^{0}$ graphs, obtained from trajectories, are distorted from the $I_{x}$ vs $\varphi_{x}$ curves obtained from perturbation theory. This difference can be seen, for example, by examining symmetric separatrix in the present Fig. 1 and comparing it with Fig. 9 of Ref. 12.

However, since the phase integral $\oint_{\mathbf{p}} \cdot d \mathbf{q}$ is a canonical invariant, ${ }^{33}$ we have

$$
\oint \mathbf{p} \cdot d \mathbf{q}=\oint \mathbf{I}^{0} \cdot d \varphi^{0}=\oint \mathbf{I} \cdot d \varphi,
$$

where the cyclic integral follows some path $C$. In the first integral we choose $C$ to be $y=$ constant, and hence in the second, $\varphi_{y}^{0}=$ constant, then in the third we deform the path (without cutting new caustics of the trajectory when they are not too distorted by the perturbation) so that $C$ is along $\varphi_{y}$ $=$ constant. We have

$$
\oint p_{x} d x=\oint I_{x}^{0} d \varphi_{x}^{0}=\oint I_{x} d \varphi_{x}=\oint I_{\alpha} d \alpha,
$$

where in the last equality we used Eq. (2.12). ${ }^{34}$ We make use of this result in the next section, where we evaluate $\oint p_{x} d x$ or $\oint I_{x}^{0} d \varphi_{x}^{0}$ from trajectory data and introduce Eq. (2.28) below for $I_{\alpha}$ into $\oint I_{\alpha} d \alpha$. In all our subsequent work, we use 
canonical invariants such as Eq. (2.20), rather than individual points on trajectories, to evaluate parameters, since the actions and angles we are using are distorted from the zeroth order actions by the perturbations, as seen in the canonical transformation formulas.

In terms of $I$ and $I_{\alpha}$ the resonance Hamiltonian is

$$
\begin{aligned}
H_{R}=I & +\lambda I_{\alpha}\left(2 I-3 I_{\alpha}\right) / 12-\frac{b}{2}\left(I_{x} I_{y}^{3} / 3\right)^{1 / 2} \cos 2 \alpha \\
& -\frac{15 a^{2}}{4}\left(I^{2}-3 I I_{\alpha}+\frac{9}{4} I_{\alpha}^{2}+\frac{I_{\alpha}^{2}}{324}\right),
\end{aligned}
$$

where $I_{x}$ and $I_{y}$ are given by Eq. (2.12).

In resonant Hamiltonians such as Eq. (2.22) it is customary $^{15}$ (e.g., Ref. 35 ) to replace the coefficient of the trigonometric term by its value at the resonance center. In the present case, we can show (see Appendix B) that the values of $I_{x}$ and $I_{y}$ at the resonant center are their average values $\bar{I}_{x}, \bar{I}_{y}$ between the pair of states that undergo the avoided crossing. Using these (constant) average actions, replacing $H_{R}$ by $E$, and defining

$$
\begin{aligned}
& \beta=\frac{2}{3} I\left(2 \lambda+135 a^{2}\right)\left(4 \lambda+135 a^{2}\right)^{-1}, \\
& q=4 b\left(\bar{I}_{x} \bar{I}_{y}^{3} / 3\right)^{1 / 2}\left(4 \lambda+135 a^{2}\right)^{-1}, \\
& C=16\left(I-15 a^{2} I^{2} / 4\right)\left(4 \lambda+135 a^{2}\right)^{-1}, \\
& D=16\left(4 \lambda+135 a^{2}\right)^{-1},
\end{aligned}
$$

Eq. (2.22) can be rearranged to give

$$
I_{\alpha}^{2}-2 \beta I_{\alpha}+2 q \cos 2 \alpha+D E-C=0
$$

which has two solutions at any energy $E$,

$$
I_{\alpha}^{(1,2)}=\beta \pm\left[C+\beta^{2}-D E-2 q \cos 2 \alpha\right]^{1 / 2} .
$$

In Eqs. (2.23)-(2.26) a term $I_{\alpha}^{2} / 324$ in Eq. (2.22), which is 729 times smaller than the $9 I_{\alpha}^{2} / 4$, was neglected. This neglect does not affect the functional form of $\mathrm{Eq}$. (2.27).

Instead of using the perturbation equations (2.23)(2.26) to evaluate the parameters in $\mathrm{Eq} .(2.27)$ we show in the next section how they may be evaluated directly from the classical trajectory data. Some comparison with the perturbation expressions is given later.

We shall later need integrals of $I_{\alpha}$ over $\alpha^{36}$ :

$$
\int_{0}^{\pi} I_{\alpha} d \alpha=\beta \pi \pm 2 \sqrt{a+2 q} \mathbf{E}[\sqrt{4 q /(a+2 q)}]
$$

when $a-2 q>0$. The function $\mathbf{E}$ is the complete elliptic integral of the second kind and

$$
a=C-D E+\beta^{2} \text {. }
$$

\section{EVALUATION OF PARAMETERS IN EQ. (2.27) FROM CLASSICAL TRAJECTORY DATA}

In this section, we first consider the primitive semiclassical quantization (Sec. A), and then evaluate the parameters in Eq. (2.27) in Sec. B.

\section{A. Primitive semiclassical quantization}

We begin by noting that since $H_{R}$ is independent of $\theta$, its canonically conjugate momentum $I$ is a constant of the motion. Its semiclassical eigenvalues are

$$
I=3 I_{x}+I_{y}=n+2 \text {, }
$$

where we have used units of $\hbar=1$ and introduced an integer $n$ given by

$$
n=3 n_{x}+n_{y} \text {. }
$$

The quantum number $n$ is actually the principal quantum number for the motion. It can be evaluated either by evaluating the phase integrals $\oint p_{x} d x$ and $\oint p_{y} d y$ separately to obtain $n_{x}$ and $n_{y}$ or by integrating the phase along the trajectory over one or more near cycles and using the "trajectory-closure" methods (in which one joins along a surface of section the end points of a near-cycle trajectory). ${ }^{37}$ We now have

$$
\begin{aligned}
& \oint\left(p_{x} d x+p_{y} d y\right)=2 \pi(n+2) . \\
& \text { trajectory }
\end{aligned}
$$

The 2 in Eqs. (3.1) and (3.3) arises from a sum of the constants in $3\left(n_{x}+\frac{1}{2}\right)$ and $\left(n_{y}+\frac{1}{2}\right)$. It can also be seen to arise by noting that a trajectory touches the caustics eight times during the near cycle-six times because of the three $x$ cycles and two times because of the $y$ cycle in the overall near cycle (cf. Fig. 6 of Ref. 12). Since each time the trajectory touches a caustic, there is a loss of phase in the primitive semiclassical wave function by $\pi / 2$, the total loss of phase due to touching the caustics in $4 \pi$. This loss corresponds to the 2 added to the main quantum number $n$ in Eq. (3.3) when the condition of single valuedness of the semiclassical wave function is imposed.

The primitive semiclassical quantization of the $\alpha$ coordinate is given, using Eq. (2.12), as Eq. (3.4) when $\alpha$ has the full $(0, \pi)$ range. ${ }^{38}$

$$
\int_{0}^{\pi} I_{\alpha} d \alpha=\int_{-\pi}^{\pi} I_{x} d \varphi_{x}=2 \pi\left(n_{x}+\frac{1}{2}\right),
$$

where the $I_{x}-\varphi_{x}$ phase integral has been evaluated at $\varphi_{y}=0$.

Using Eqs. (2.28) and (3.4) it can be shown (Appendix C) that the two primitive semiclassical eigenvalues cross, rather than avoid each other.

\section{B. Evaluation of the parameters in the resonance equation (2.27) from trajectory data}

We consider a series of trajectory calculations performed at a given $I$. To find $q$ at the crossing point (or indeed any $\lambda$ ) and at the given $I$, the separatrix of the motion at that $\lambda$ and $I$ will be used. The separatrix is that trajectory which separates the two kinds of motion possible in the $\left(I_{\alpha}, \alpha\right)$ plane, namely "rotation" and "libration," as seen in Fig. 1, and indeed also separates the rotational trajectories above the separatrix from the rotational trajectories below it.

On the separatrix $\left(I_{\alpha}-\beta\right)^{2}$ vanishes at $\alpha=0$ and $\alpha=\pi$. Thereby, the energy of the separatrix at any $\lambda$ is seen from $\mathrm{Eq}$. (2.27) to be

$$
E_{S}=\left(C+\beta^{2}-2 q\right) / D \text {. }
$$

At that energy, Eq. (2.28) yields the two branches of the separatrix:

$$
I_{\alpha}^{(1,2)}=\beta \pm 2 q^{1 / 2} \sin \alpha .
$$


The separatrix is "self-crossing" at $a=0$ and $\pi$. A Poincaré surface of section in the $\left(I_{x}, \varphi_{x}\right)$ plane can be obtained by taking a cut through the trajectory on the plane $y=0$. This choice of $\varphi_{y}$ corresponds to $2 \alpha=\varphi_{x}+\pi$, and the two branches (3.6) are transformed to Eq. (3.7), on using Eq. (2.12) for $I_{\alpha}$.

$$
I_{x}^{(1,2)}=\beta / 2 \pm q^{1 / 2} \cos \left(\varphi_{x} / 2\right)
$$

resulting in a surface of section plot similar to the curves $(b)$, (c) of Fig. 1. The area enclosed between the two branches is

$$
\begin{aligned}
S & =\int_{0}^{\pi}\left[I_{\alpha}^{(1)}-I_{\alpha}^{(2)}\right] d \alpha \\
& =\int_{-\pi}^{\pi}\left[I_{x}^{(1)}-I_{x}^{(2)}\right] d \varphi_{x}=8 q^{1 / 2} .
\end{aligned}
$$

Hence, $q$ can be determined from

$$
q=S^{2} / 64 \text {. }
$$

This equation applies, incidentally, regardless of whether $\lambda$ is at the avoided crossing or not. In trajectory calculations, $S$ can be determined as follows: when the separatrix has been found, a Poincaré surface of section in the $\left(p_{x}, x\right)$ plane is drawn (for an example, see Fig. 8 of Ref. 12). The area $S$ enclosed by the branches of the separatrix is the difference between areas of the two elliptic curves [using Eq. (2.21)]. ${ }^{39}$ have

We next determine $\beta$. From Eqs. (2.28) and (2.21) we

$$
2 \beta \pi=\int_{0}^{\pi}\left[I_{\alpha}^{(1)}(E)+I_{\alpha}^{(2)}(E)\right] d \alpha=\int_{-\pi}^{\pi}\left(I_{x}^{(1)}+I_{x}^{(2)}\right) d \varphi_{x},
$$

where the two $\varphi_{x}$ integrals can also be obtained from the $\oint p_{x} d x$ phase integrals for the pair of trajectories [Eq. (2.21)]. The integrals are calculated at the same $I$ and at the same $E$, e.g., an $E$ in the vicinity of $E^{(1)}$ and $E^{(2)}$. At the avoided crossing Eqs. (3.10) and (3.4) yield

$$
\beta=n_{x}^{(1)}+n_{x}^{(2)}+1 \text { (at the avoided crossing), }
$$

where $n_{x}^{(1,2)}$ are the $x$-quantum numbers (integers) of the primitive semiclassical pair of states involved in the crossing.

To obtain $C$ and $D$ at any $\lambda$ we can use two integrals derived from Eq. (3.4), knowing $q$ and $\beta$ at that $\lambda$, for two values of $E$ and hence of $n_{x}$ :

$$
\begin{aligned}
\int_{0}^{\pi} I_{\alpha} d \alpha & =\beta \pi \pm \int_{0}^{\pi}\left[C+\beta^{2}-D E-2 q \cos 2 \alpha\right]^{1 / 2} d \alpha \\
& =2 \pi\left[n_{x}+\frac{1}{2}\right] .
\end{aligned}
$$

When the system is not at an avoided crossing, the two $n_{x}$ 's can be simply chosen to be the $n_{x}$ 's of the pair of states under consideration. Equation (3.12) applies regardless of whether or not one uses eigentrajectories (i.e., trajectories with integer $\left.n_{x}^{(1,2)}\right) . E^{(1,2)}$ are primitive semiclassical energies of the two states. The integral (3.12) is expressed in terms of an elliptic integral, as in Eq. (2.29), thereby leading to two transcendental equations for $C$ and $D$ in terms of the known quantities $\beta, q, n_{x}^{(1,2)}$, and $E^{(1,2)}$. In an iterative scheme, a first approximation for $C$ and $D$ may be obtained by neglecting $q$ :

$$
\left[2 n_{x}+1\right]^{2}-2 \beta\left[2 n_{x}+1\right]+D E-C \cong 0 \text {. }
$$

The two equations in Eq. (3.12) for $E^{(1)}$ and $E^{(2)}$ are not distinct at the $\lambda$ where there is an avoided crossing, as was shown in Appendix C. In this case one applies Eq. (3.12) or
(3.13) using data at noninteger $n_{x}$ 's. For example, Eq. (3.13) yields for $D$,

$$
D \cong-8\left(d^{2} E / d n_{x}^{2}\right)^{-1} \text {. }
$$

With that value of $D, \mathrm{Eq}$. (3.13) yields a zeroth order value for $C$. These values can be refined by iteration, using the rigorous expression (3.12) to give improved values of $C$ and $D$ due to the neglect of $q$ was less than $1 \%$ at $b=0.005$ and around $6 \%$ at $b=0.02 .{ }^{40}$ An error of this size in $D$ causes a similar error in the calculation of splitting at the avoided crossing, which is minor.

In evaluating $C$ and $D$ from Eq. (3.13) or (3.14) it is desirable to use trajectories which are not too close to the separatrix, for then the correction due to the $q$ term in Eq. (3.12) is less.

We note in passing that the perturbation expressions $(2.23)-(2.26)$ yield $q / D$ and $C / D$ ratios which are independent of $\lambda$, and so this feature could be used to obtain these constants away from the crossing, knowing their values at the crossing.

\section{UNIFORM SEMICLASSICAL QUANTIZATION}

We denote the two dimensional primitive semiclassical wave function of our system in the $\left(I_{x}, I_{y}, \varphi_{x}, \varphi_{y}\right)$ actionangle variables by $\Psi^{\prime}(\varphi)$. Single valuedness of the quantum mechanical or semiclassical wave function yields the following periodicity result:

$$
\Psi^{\prime}\left(\varphi_{x}+2 \pi k, \varphi_{y}+2 \pi l\right)=\Psi^{\prime}\left(\varphi_{x}, \varphi_{y}\right)
$$

The primitive semiclassical wavefunction is equal to, ${ }^{41}$ apart from a preexponential factor (the van Vleck determinant),

$$
\Psi^{\prime}\left(\varphi_{x}, \varphi_{y}\right) \sim \exp \left\{i\left[\int^{\varphi_{x}}\left(I_{x}-\frac{1}{2}\right) d \varphi_{x}+\int^{\varphi_{y}}\left(I_{y}-\frac{1}{2}\right) d \varphi_{y}\right]\right\}
$$

The $\frac{1}{2}$ 's in Eq. (4.2) ensure that the semiclassical wave function satisfies the correct periodicity property in $\mathrm{Eq}$. (4.1): an increase of phase of $\varphi_{x}$ by $2 \pi$, for example, increases the phase of $\Psi^{\prime}$ by $\oint\left(I_{x}-\frac{1}{2}\right) d \varphi_{x}$, and since $\oint I_{x} d \varphi_{x}$ equals $2 \pi\left(n_{x}+\frac{1}{2}\right)$ in the present case, with $n_{x}$ being an integer, the phase of $\Psi^{\prime}$ in Eq. (4.2) changes by $2 \pi n_{x}$. Hence, this $\Psi^{\prime}$ given by Eq. (4.2) satisfies Eq. (4.1).

We next make the following substitution in Eq. (2.27):

$$
I_{x} \rightarrow \frac{1}{i} \frac{\partial}{\partial \varphi_{x}}+\frac{1}{2}, \quad I_{y} \rightarrow \frac{1}{i} \frac{\partial}{\partial \varphi_{y}}+\frac{1}{2} .
$$

The $\frac{1}{2}$ permits this substitution, in conjunction with the wave function (4.2), to yield, on differentiation, the original classical Eq. (2.27) in terms of $I_{x}$ and $I_{y}$.

If we make the canonical transformation (2.12), the periodicity condition (4.1) yields

$$
\Psi[\alpha+(k-3 l) \pi, \theta+2 \pi l]=\Psi(\alpha, \theta),
$$

where $\psi$ is numerically equal to $\Psi^{\prime}$ at any point in angle space. Since $I$ is a constant of the motion and $\theta$ a cyclic variable, this total wave function $\Psi$ can immediately be factorized as

$$
\Psi(\alpha, \theta)=\psi(\alpha) G(\theta) .
$$

Further, one can replace $k-3 l$, an integer, by another in- 
teger $m$. Thus

$$
\psi(\alpha+m \pi)=\psi(\alpha) .
$$

All such functions automatically satisfy

$$
\psi(\alpha+\pi)=\psi(\alpha)
$$

regardless of the value of $m$. We will use this periodicity condition in solving the Schrödinger equation corresponding to our resonance Hamiltonian obtained from Eqs. (2.27) and (4.3).

The primitive semiclassical form of $\Psi(\alpha, \theta)$ is obtained by expressing the $I_{x}, I_{y}, \varphi_{x}$ and $\varphi_{y}$ in Eq. (4.2) in terms of the $\alpha$ and $\theta$ variables. Using Eq. (2.12) one finds (apart from the van Vleck preexponential factor)

$\Psi(\alpha, \theta) \sim \exp \left\{i\left[\int^{\alpha}\left(I_{\alpha}-1\right) d \alpha+\int^{\theta}(I-2) d \theta\right]+i \frac{\pi}{2}\right\}$

Since $I$ is a constant of the motion, one sees from a comparison with Eq. (4.5) that

$$
\psi(\alpha) \sim \exp \left\{i \int^{\alpha}\left(I_{\alpha}-1\right) d \alpha\right\}
$$

[The constant phase factor of $+i \pi / 2$ in Eq. (4.8) can be omitted without loss of generality.]

To obtain the semiclassical energy levels in the form of a uniform approximation, Eq. (2.27) is next converted into a differential equation by replacing the actions by differential operators. The resulting differential equation is one dimensional because $I$ can be replaced by its constant value (3.1). The choice ${ }^{4 I}$

$$
I_{\alpha} \rightarrow \frac{1}{i} \frac{d}{d \alpha}+1
$$

is consistent with the wave function (4.9), because one obtains the original classical equation (2.27) upon introducing Eq. (4.10) into Eq. (2.27) and operating on the wave function (4.9).

The replacement transforms Eq. (2.27) into a Schrödinger equation for $\psi(\alpha)$ :

$$
\frac{d^{2} \psi}{d \alpha^{2}}-2 i(\beta-1) \frac{d \psi}{d \alpha}+(A-2 q \cos 2 \alpha) \psi=0
$$

with

$$
A=C-D E+2 \beta \text {. }
$$

If we solve Eq. (4.11) for an auxiliary function $F(\alpha)$,

$$
F(\alpha)=\exp [i(1-\beta) \alpha] \psi(\alpha)
$$

instead of $\psi$, we find that Eq. (4.11) transforms into a Mathieu equation ${ }^{42,43}$

$$
\frac{d^{2} F}{d \alpha^{2}}+\left(a_{v}-2 q \cos 2 \alpha\right) F=0
$$

where

$$
a_{v}=C-D E+\beta^{2} .
$$

One sees from Eq. (4.13) that $F$ is a complex exponential function of $\alpha$ multiplied by another function of $\alpha$ which has period $\pi$. Indeed, this condition is also the usual condition onMathieu functions of fractional order, so that our desired function $F$ is such a function.
In common with other problems that involve nonlinear resonances, we have obtained a pendulum ("hindered rotor," "restricted rotator") Hamiltonian, 15 apart, in Eq. (4.11), from the term containing $d \psi / d \alpha$. In physical problems involving hindered rotors, ${ }^{44} 2 q$ is the barrier height for internal rotation.

The solution of Eq. (4.14) can be expressed in terms of the Floquet solution

$$
F_{v}(\alpha)=e^{i v \alpha} P(\alpha),
$$

where $P(\alpha)$ has the period $\pi$ and $v$, the characteristic exponent (or the order of the solutions of the Mathieu equation), will be determined below. In terms of this Floquet solution, the wave function $\psi(\alpha)$ can be rewritten as

$$
\psi(\alpha)=\exp [i(v+\beta-1) \alpha] P(\alpha) .
$$

In order to determine $v$ in terms of $\oint I_{x} d \varphi_{x}$ phase integrals, we increase $\varphi_{x}$ by $2 \pi$ while leaving $\varphi_{y}$ the same. The semiclassical wave function (4.2) changes then as

$$
\Psi^{\prime}\left(\varphi_{x}+2 \pi, \varphi_{y}\right)=\exp \left[i \int_{0}^{2 \pi}\left(I_{x}-\frac{1}{2}\right) d \varphi_{x}\right] \Psi^{\prime}\left(\varphi_{x}, \varphi_{y}\right)
$$

When $\varphi_{x}$ increases by $2 \pi$ at constant $\varphi_{y}, \alpha$ undergoes the concurrent change of $\pi$. Equation (4.7) yields

$$
\psi(\alpha+\pi)=\exp [i(v+\beta-1) \pi] \psi(\alpha) .
$$

Similarly, $G(\theta)$ in Eq. (4.5) stays constant since $\theta=\varphi_{y}$ [Eq. (2.12)] and $\varphi_{y}$ is being held constant. Thus $\Psi(\alpha+\pi, \theta)$ obeys

$$
\Psi(\alpha+\pi, \theta)=\exp [i(v+\beta-1) \pi] \Psi(\alpha, \theta) .
$$

By comparing Eqs. (4.18) and (4.20) we obtain

$$
(\nu+\beta) \pi=\int_{0}^{2 \pi} I_{x} d \varphi_{x} .
$$

If we recall that the phase integral is $2 \pi\left(n_{x}+\frac{1}{2}\right)$, then

$$
v=2 n_{x}+1-\beta \text {. }
$$

The characteristic exponent in $v$ is fractional in general. Expansions of the characteristic value (eigenvalue) $a_{v}$ of Eq. (4.14) in terms of $q$ and $v$ exist for noninteger $v$. (We used that in page 20 of Ref. 42.) When $v$ is an integer $r$, it is convenient to associate $v=r$ with the characteristic value $a_{r}(q)$ and $v=-r$ with $b_{r}(q)$.

Uniform semiclassical eigenvalues $E$ are then found from the $a_{v}$ 's using Eq. (4.15). Thereby the relation

$$
E=\left(C-a_{v}+\beta^{2}\right) D^{-1}
$$

completes the quantization scheme. The values of the parameters $C, D, q$, and $\beta$ are evaluated as in Sec. III B. The $a_{v}$ 's in Sec. VI were determined from expansions ${ }^{42}$ in powers of $q$ where $q$ was small and by interpolation from tables ${ }^{45}$ when $q$ was large.

A pair of states with quantum numbers $n_{x}^{(1)}$ and $n_{x}^{(2)}$ approach each other in energy most closely when $v^{(1)}$ and $v^{(2)}$, as calculated from Eq. (4.22), are integers such that

$$
v^{(1)}=-v^{(2)} \text {. }
$$

This condition is fulfilled whenever

$$
\beta=n_{x}^{(1)}+n_{x}^{(2)}+1 \text {. }
$$

Comparison of Eq. (4.25) with Eq. (3.11) shows that the cor- 
responding minimum splittings in energy are the splittings at the avoided crossing. They can be calculated by

$$
\Delta E=E^{(1)}-E^{(2)}=\left(a_{v}-b_{v}\right) / D .
$$

In particular, for small $q$,

$$
\begin{aligned}
& \Delta E_{1}=\left(\alpha_{1}-b_{1}\right) / D=2 q / D+O\left(q^{3}\right), \\
& \Delta E_{2}=\left(a_{2}-b_{2}\right) / D=q^{2} / 2 D+O\left(q^{4}\right), \\
& \Delta E_{3}=\left(a_{3}-b_{3}\right) / D=q^{3} / 32 D+O\left(q^{5}\right) .
\end{aligned}
$$

In the present case, these are the splittings for the pair of states $\left(n_{x}, n_{y}\right)=[(1,0),(0,3)],[(2,0),(0,6)]$, and $[(3,0),(0,9)]$, respectively.

\section{WIDTHS OF RESONANCES AND SPLITTINGS OF QUANTAL ENERGY LEVELS}

In standard Chirikov resonance theory, ${ }^{15}$ a Hamiltonian containing a classical resonance can be expanded as a quadratic function of $I_{\alpha}$ around the resonance center $I_{\alpha}^{r}$ as (using the notation of Ref. 13)

$$
H=H_{0}\left(\mathbf{I}^{r}\right)-\frac{1}{2} \Omega\left(I_{\alpha}-I_{\alpha}^{r}\right)^{2}+A\left(I_{x}^{r}, I_{y}^{r}\right) \cos \mathrm{k} \alpha,
$$

$k$ is an integer. The coefficient of the trigonometric term is evaluated at the center of the nonlinear resonance defined in Appendix B. The vertical extrema of the separatrix of $I_{\alpha}$ vs $\alpha$ are $\pm 2(A / \Omega)^{1 / 2}$, thus leading to a full width $W$ in $I_{\alpha}$ space of $4(A / \Omega)^{1 / 2}$. The maximum $I_{\alpha}$ in Eq. (5.1) occurs at $k \alpha=\pi$, whereas that in Eq. (2.27) occurs at $2 \alpha=0$. This difference can be removed by replacing the $k \alpha$ in Eq. (5.1) by $k \alpha+\pi$. Further comparison then shows that $A, \Omega, I_{a}^{r}$, and $k$ are equal to $2 q, 2, \beta$, and 2 . The classical resonance width $4(A / \Omega)^{1 / 2}$ then becomes

$$
W=4 q^{1 / 2} \text {. }
$$

The difference in $\alpha$-quantum numbers $n_{\alpha}$ for the pair of states undergoing the avoided crossing, $n_{\alpha}^{(1)}-n_{\alpha}^{(2)}$, is seen from the relation between $I_{\alpha}$ and $I_{x}$ in Eq. (2.12) to be $2\left(n_{x}^{(1)}-n_{x}^{(2)}\right)$. One might expect that whenever this difference exceeds the resonance width $W$, i.e., whenever

$$
\left|n_{x}^{(1)}-n_{x}^{(2)}\right|>2 q^{1 / 2}
$$

the splitting at the point of closest approach of quantum mechanical energy levels of the two states undergoing the avoided crossing is small. We give later in Table IV a comparison of both sides of Eq. (5.3), and also give the splitting.

\section{CALCULATIONS}

In this section, we compare energy eigenvalues obtained by quantum, primitive semiclassical, uniform semiclassical, and perturbation methods. In particular, we use the uniform expression Eq. (4.23) and show how it can be used to improve the primitive semiclassical eigenvalues. In all these calculations, we have taken $a=0.02$.

\section{A. Quantum results}

In their recent comparison of quantal, classical, and semiclassical behavior at an avoided crossing, Noid et al. ${ }^{12}$ examined perturbations of the $\left(n_{x}, n_{y}\right)=(1,0)$ and $(0,3)$ pair of levels which show an avoided crossing near $\lambda=0.055$ when $b=0.005$. A plot of their quantum mechanical eigen-

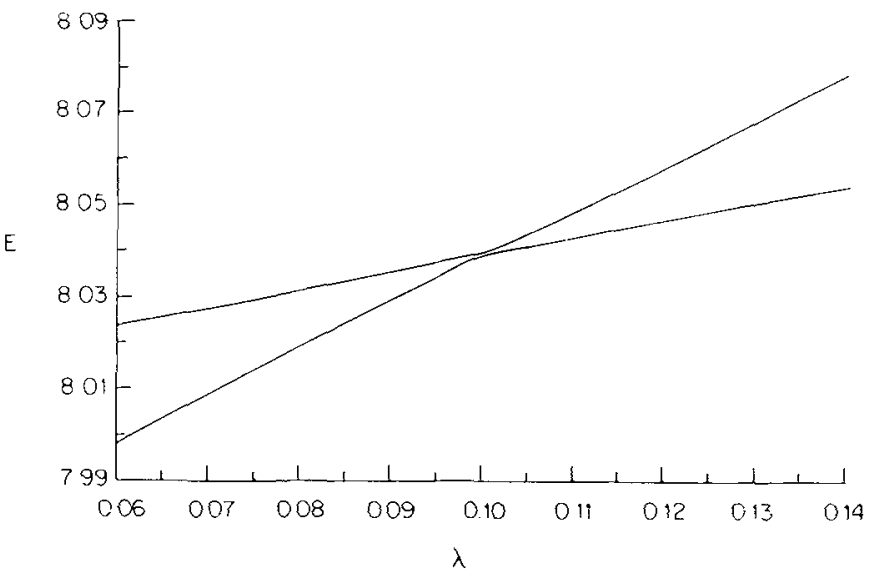

FIG. 2. Plot of eigenvalues of the pair of states $(2,0)$ and $(0,6)$ vs perturbation parameter $\lambda$ for $b=0.005$.

values vs $\lambda$ appears in Fig. 1 of Ref. 12. Plots for other conditions are given in their Figs. 2 and 3.

We extended their calculations to several other pairs of states that show avoided crossings. A 400 element basis set of harmonic oscillator wavefunctions was used and the resulting matrices were diagonalized using the EISPACK matrix diagonalization package. ${ }^{46}$ The eigenvalues $E_{Q}$ in Table I so obtained were accurate to one part in $10^{4}$. A plot of the pair $[(2,0),(0,6)]$ at $b=0.005$ is given in Fig. 2 and, for comparison, the pair $[(1,0),(0,3)]$ of Ref. 12 at $b=0.005$ is given in Fig. 3. For brevity, we have omitted a pair $[(1,2),(0,5)]$ which shows an avoided crossing near $\lambda=-0.11$, a pair $[(1,1),(0,4)]$ which gave parallel (i.e., nonapproaching) curves, both at $b=0.005$, and the pair $[(2,0),(1,3)]$ which shows an avoided crossing near $\lambda=0.013$.

\section{B. Primitive semiclassical trajectory eigenvalues}

Primitive semiclassical eigenvalues were obtained by quantizing classical trajectories. ${ }^{23}$ Trajectories were obtained by integrating the equations of motion for the Hamiltonian (2.8) using the DEROOT program ${ }^{47}$ Poincare surface of section results were recorded for $p_{x}$ vs $x$ every time the trajectory crossed the line $y=0$ in a particular direction (e.g., $y>0$ ). The evaluation of the area enclosed by the sur-

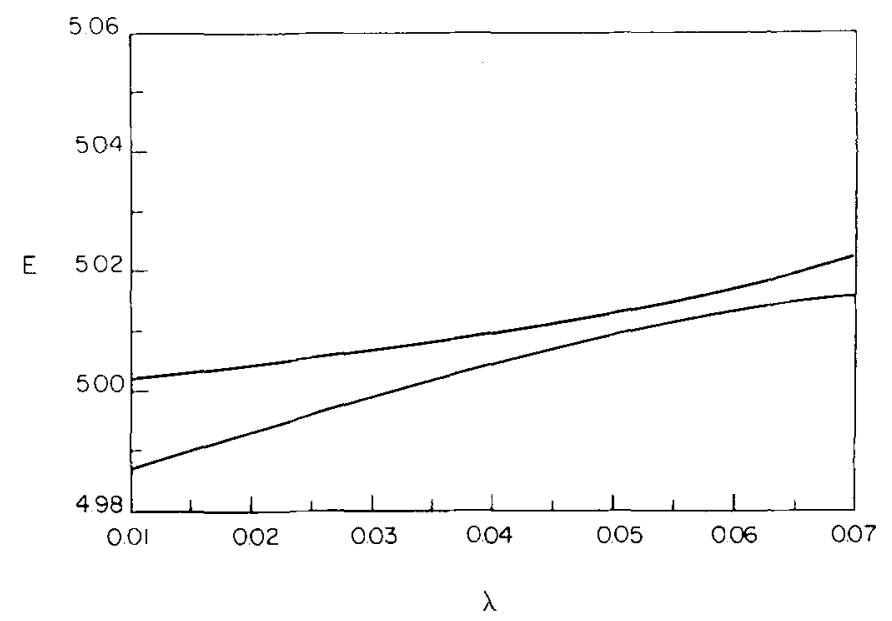

FIG. 3. Same as Fig. 2, except for pair $(1,0),(0,3)$. 
TABLE I. Comparison of quantum with primitive semiclassical trajectory and perturbation eigenvalues for the $(2,0)(0,6)$ pair of states, $b=0.005, a=0.02$.

\begin{tabular}{|c|c|c|c|c|c|c|}
\hline \multirow[b]{2}{*}{$\lambda$} & \multicolumn{2}{|c|}{$E_{Q}{ }^{2}$} & \multicolumn{2}{|c|}{$E_{\mathrm{PSCT}}{ }^{\circ}$} & \multicolumn{2}{|c|}{$E_{\mathrm{PSCP}}$} \\
\hline & $(2,0)^{d}$ & $(0,6)^{d}$ & $(2,0)$ & $(0,6)$ & $(2,0)$ & $(0,6)$ \\
\hline 0.06 & 8.0237 & 7.9981 & 8.0239 & 7.9983 & 8.0243 & 8.0014 \\
\hline 0.07 & 8.0276 & 8.0086 & 8.0279 & 8.0087 & 8.0286 & 8.0123 \\
\hline 0.08 & 8.0315 & 8.0189 & 8.0316 & 8.0191 & 8.0328 & 8.0231 \\
\hline 0.10 & 8.0394 & 8.0392 & 8.0395 & 8.0394 & 8.0411 & 8.0448 \\
\hline 0.12 & 8.0469 & 8.0593 & 8.0473 & 8.0594 & 8.0495 & 8.0665 \\
\hline 0.14 & 8.0544 & 8.0788 & 8.0548 & 8.0784 & 8.0579 & 8.0881 \\
\hline
\end{tabular}

a Full quantum calculation using a basis of 400 harmonic oscillator states.

${ }^{\mathrm{b}}$ Primitive semiclassical calculation using trajectories.

' Primitive semiclassical calculation using perturbation theory.

'These labels are not meaningful in the avoided crossing region.

face of section can be evaluated more conveniently with the aid of the action-angle plot $I_{x}^{0}$ vs $\varphi_{x}^{0}$. When evaluating the area $\oint I_{x}^{0} d \varphi_{x}^{0}$, the variables involved were deduced from the $\left(x, p_{x}\right)$ set by usual transformation (2.9), to which should be added 0 superscripts. Similarly, we calculated the area $\oint I_{y}^{0} d \varphi_{y}^{0}$ for $x=0$. These integrals are set equal to $2 \pi\left(n_{x}+\frac{1}{2}\right)$ and $2 \pi\left(n_{y}+\frac{1}{2}\right)$, respectively, and the initial conditions of the trajectory were varied until the $n_{x}$ and $n_{y}$ are the desired integers. The results for the $[(2,0),(0,6)]$ pair are given in Table I. As can be seen from Table I, the primitjve semiclassical eigenvalues again agree very well with the quantum results for all $\lambda$ except, of course, near the avoided crossing which we did not examine for these data. This aspect had been extensively investigated in Ref. 9 for the data shown in Figs. 1 and 3 there. As shown in that article, the primitive semiclassical eigenvalues cross instead of avoiding each other.

The last column in Table I gives a comparison between quantum and primitive semiclassical trajectory eigenvalues and the primitive semiclassical perturbation eigenvalues obtained from Eqs. (2.23)-(2.26), (2.29), and (3.4), iterating the latter to obtain the $E$ 's. Theoretical arguments given in Appendix $\mathrm{C}$ show that the primitive semiclassical eigenvalues should cross instead of avoiding each other. When compared with the quantum ones, the trajectory values are seen to be superior to the perturbation ones.

\section{Uniform semiclassical trajectory treatment}

We next apply the uniform scheme of the Sec. IV to obtain the splitting at the avoided crossing. The characteristic exponent (orders of the Mathieu function) for the pair of states are obtained from Eqs. (4.22), (4.24), and (4.25). For example, for the $(1,0)$ and $(0,3)$ states, denoted by $(1)$ and $(2)$, respectively, one sees from Eq. (4.25) that $\beta$ is 2 at the avoid- ed crossing, and hence from Eq. (4.22) that $v^{(1)}$ and $v^{(2)}$ equal +1 and -1 , respectively. Similarly, for the $(2,0)$ and $(0,6)$ pair, they equal +2 and -2 , respectively. Thus, the differences $\left(a_{1}-b_{1}\right) / D$ and $\left(a_{2}-b_{2}\right) / D$ yield the splittings at the respective avoided crossing for the two pairs. In the case of $D$, the second derivative in Eq. (3.14) was calculated as follows for a $(n, 0),(0,3 n)$ pair. Typically, first derivatives were calculated from $E$ 's at $n_{x}$ 's equal to $(n-0.01$ and $n)$ and to $(n, n+0.01)$ and averaged. They were also calculated from $n_{x}$ 's equal to $(-0.01,0)$ and to $(0,0.01)$ and again averaged. This pair of first derivatives then yielded a second derivative. We had to use $n_{x}$ 's not near the separatrix, which occurred at an $n_{x}$ intermediate between 0 and $n$.

The values of $S, q$, and $D$ calculated at the avoided crossing point are given in Table II, together with the splitting $\Delta E_{\mathrm{USC}}$ calculated from the values of $a_{r}-b_{r}$ using Eq. (4.26), and with the quantum values $\Delta E_{Q}$.

\section{Semiclassical perturbation treatment}

In order to see the improvements provided by the uniform formula, we have made some comparisons between primitive and uniform semiclassical results, using the perturbation expressions $(2.23)-(2.26)$ for the parameters. To obtain $q$ in Eq. (2.24) one needs the average actions $\bar{I}_{x}$ and $\bar{I}_{y}$. They are

$$
\begin{aligned}
& \bar{I}_{x}=\left[I_{x}^{(1)}+I_{x}^{(2)}\right] / 2=\frac{1}{2}\left(n_{y}^{(1)}+n_{y}^{(2)}+1\right), \\
& \bar{I}_{y}=\left[I_{y}^{(1)}+I_{y}^{(2)}\right] / 2=\frac{1}{2}\left(n_{x}^{(1)}+n_{x}^{(2)}+1\right) .
\end{aligned}
$$

$v^{(1)}$ and $v^{(2)}$ for the $[(1,0),(0,3)]$ pair are the same as in Eq. (4.22) with $\beta$ now being found using the known values of the perturbation parameters in Eq. (2.23).

TABLE II. Splitting $\Delta E$ and other properties at an avoided crossing $\lambda=\lambda_{c}$.

\begin{tabular}{lllllll}
\hline \hline States & $b$ & $S\left(\lambda_{c}\right)$ & $q\left(\lambda_{c}\right)$ & $D\left(\lambda_{c}\right)^{\mathrm{a}}$ & $\Delta E_{\text {USC }}$ & $\Delta E_{Q}$ \\
\hline$(1,0)(0,3)$ & 0.005 & 2.73 & 0.117 & 60 & $3.9 \times 10^{-3}$ & $3.3 \times 10^{-3}$ \\
$(2,0)(0,6)$ & 0.005 & 3.24 & 0.164 & 36 & $3.8 \times 10^{-4}$ & $1.7 \times 10^{-4}$ \\
$(1,0)(0,3)$ & 0.03 & 4.81 & 0.362 & 43 & $1.7 \times 10^{-2}$ & $1.9 \times 10^{-2}$ \\
\hline \hline
\end{tabular}

"Estimated from $d^{2} E / d n_{x}^{2}$ using Eq. (3.14). 
TABLE III. Comparison of primitive and uniform semiclassical eigenvalues (perturbation theory).

\begin{tabular}{|c|c|c|c|c|c|}
\hline$b$ & $\lambda$ & $(1,0)$ & $(0,3)$ & $(1,0)^{\mathrm{c}}$ & $(0,3)^{\mathrm{c}}$ \\
\hline 0.005 & $\begin{array}{l}0.01 \\
0.03 \\
0.05 \\
0.055 \\
0.060 \\
0.080\end{array}$ & $\begin{array}{l}\text { a } \\
5.007 \\
5.012 \\
5.013 \\
5.015 \\
5.020\end{array}$ & $\begin{array}{l}4.987 \\
4.999 \\
5.011 \\
5.014 \\
5.016 \\
5.028\end{array}$ & $\begin{array}{l}5.003 \\
5.008 \\
5.009 \\
5.012 \\
5.013 \\
5.019\end{array}$ & $\begin{array}{l}4.987 \\
4.999 \\
5.009 \\
5.012 \\
5.014 \\
5.029\end{array}$ \\
\hline 0.03 & $\begin{array}{l}0.01 \\
0.04 \\
0.07 \\
0.10 \\
0.13\end{array}$ & $\begin{array}{l}\mathrm{a} \\
\mathrm{a} \\
5.014 \\
5.023 \\
5.031\end{array}$ & $\begin{array}{l}\mathrm{b} \\
\mathrm{b} \\
5.016 \\
5.036 \\
5.054\end{array}$ & $\begin{array}{l}5.012 \\
5.021 \\
5.008 \\
5.018 \\
5.027\end{array}$ & $\begin{array}{l}4.985 \\
4.996 \\
5.033 \\
5.048 \\
5.063\end{array}$ \\
\hline
\end{tabular}

${ }^{a}$ In these cases the smallest value of the integral (3.4) exceeded $3 \pi$ and so a rotational-type integral could not be used. In all cases $I_{x}>I / 3$ at places.

' In these cases, the largest value of the integral (3.4) was less than $\pi$, and so a rotational type integral could not be used. $I_{x}$ was greater than $I / 3$ at many places.

${ }^{c}$ These labels are not meaningful in the avoided crossing region.

In examining the $I_{x}$ vs $\varphi_{x}$ plots from Eqs. (2.17) and (2.27) we found it convenient to plot $\varphi_{x}$ as a function of $I_{x}$, rather than the reverse. The primitive semiclassical energies are obtained from Eqs. (2.29) and (3.4), and the uniform values are obtained from Eq. (4.23). The results are given in Table III.

The relation between semiclassical and quantum mechanical perturbation theory for the splitting of pairs of states $\left[\left(v_{x}, v_{y}\right),\left(v_{x}-1, v_{y}+3\right)\right]$ was discussed in Ref. 12, using first order degenerate perturbation theory. The $[(2,0),(0,6)]$ pair belongs instead to the class $\left[\left(v_{x}, v_{y}\right)\right.$, $\left.\left(v_{x}-2, v_{y}+6\right)\right]$ and requires second order perturbation theory since the off-diagonal matrix elements directly connecting the two states are zero. We have treated this system, and the results are more or less comparable to those obtained from the uniform semiclassical perturbation theory. We omit giving the details, since the main focus of the present paper is to show how to convert the trajectory data to uniform semiclassical eigenvalues.

\section{E. Comparison of widths and splittings}

In Table IV we compare the two sides of Eq. (5.3) for a series of pairs of states $(m, 0),(0,3 m)$ for $b=0.005$. Here $\left|n_{x}^{(1)}-n_{x}^{(2)}\right|$ equals $m$. (For simplicity we have used perturbation theory to illustrate the comparison between widths and $m$.) For the pairs of states $(m, 0)(0,3 m)$ it is established by Eq. (4.27) that their splittings vary as $q^{m}$ when $q$ is small. There- fore the correlation between condition (5.3) and small level splittings is evident. This connection can be corroborated further by increasing $b$ and thereby $q$ [Eq. (2.24)], and calculating the splitting of the quantum levels. The width of the resonance and the splitting at avoided crossing both increase, as can be seen both by perturbation theory and by the data in Table II.

\section{DISCUSSION}

We have seen that in the uniform semiclassical theory of avoided crossings the pair of states do indeed avoid each other, whereas the primitive semiclassical ones cross. As discussed in Ref. 12, one of the principal approximations is the replacement of the Fourier coefficient of $\cos 2 \alpha$ by its "average" or "resonance" value. It leads to poorer agreement between quantum and semiclassical values for the case where the splittings are associated with large changes of quantum numbers. Other researchers in the field have encountered the same problem. In their recent work on the local modes of ABA triatomic molecules ${ }^{35,48}$ Sibert et al. used that approximation to calculate splittings between pairs of local modes. Their semiclassical results agreed well with quantum mechanical calculations when the splittings were large. The discrepancies were considerable, however, for small splittings, associated with large variations in $\left|I_{i}-I_{i}^{r}\right|$ (e.g., the $|4,0\rangle \pm|0,4\rangle$ and $|5,0\rangle \pm|0,5\rangle$ pairs of local modes in Table II of Ref. 48).

TABLE IV. Comparison of resonance widths ${ }^{\mathrm{a}, \mathrm{b}}$ and energy splittings ${ }^{\mathrm{a}}$.

\begin{tabular}{lcccccccc}
\hline \hline & $\begin{array}{c}\lambda \text { at avoided } \\
\text { Pair of states }\end{array}$ & crossing & $\left|n_{x}^{(1)}-n_{x}^{(2)}\right|$ & \multicolumn{3}{c}{ For $b=0.005$} & \multicolumn{3}{c}{ For $b=0.03$} \\
& & & $W$ & $\Delta E$ & $q$ & $W$ & $\Delta E$ \\
\hline$(1,0),(0,3)$ & 0.0540 & 1 & 0.121 & 1.39 & 0.0041 & 0.726 & 3.40 & 0.0227 \\
$(2,0),(0,6)$ & 0.0945 & 2 & 0.214 & 1.85 & $6 \times 10^{-4}$ & 1.284 & 4.53 & 0.0194 \\
$(3,0),(0,9)$ & 1.1350 & 3 & 0.307 & 2.22 & $6 \times 10^{-5}$ & 1.842 & 5.44 & 0.0071 \\
$(4,0),(0,12)$ & 0.1755 & 4 & 0.400 & 2.53 & $1 \times 10^{-6}$ & 2.400 & 6.20 & 0.0016 \\
$(5,0),(0,15)$ & 0.2160 & 5 & 0.493 & 2.81 & $2 \times 10^{-8}$ & 2.958 & 6.88 & 0.0002 \\
\hline \hline
\end{tabular}

a Calculated from perturbation theory.

${ }^{b}$ Classical resonance width defined by Eq. (5.2). The right-hand side of Eq. (5.3) is $W / 2$. 
We see from Tables II and III that the uniform semiclassical theory supplements the primitive semiclassical theory in two respects: (1) it produces the avoided crossing, (2) it permits the calculation of eigenvalues in other cases where primitive semiclassical theory fails (or perhaps requires an alternative procedure). Interestingly enough, three of the five cases in Table III [those under $(1,0)$ ] for which primitive semiclassical perturbation eigenvalues were not obtained were also not obtained from trajectories in Ref. 12 using the simple quantization employed there.

We note in passing that uniform semiclassical theory has been used, in conjunction with perturbation theory, to treat the Hénon-Heiles problem. ${ }^{49}$ In that case, the linear term in Eq. (2.27), which plays an important role in our system was either absent or not discussed.

The primitive semiclassical eigenvalue plots cross and can be regarded as the diabatic curves, while the uniform semiclassical and quantum plots are the adiabatic curves. A remarkable feature of the results, therefore, is that the diabatic curves can be generated using the full Hamiltonian, provided one calculates them in a primitive semiclassical way. Customarily, diabatic curves are obtained by neglecting some term or terms in the Hamiltonian.

\section{CONCLUDING REMARKS}

In this article, we have shown that a classical resonance leads to an avoided crossing and we have presented a uniform semiclassical theory of such avoided crossings. The treatment involves obtaining a functional form for the $\mathrm{Ha}$ miltonian by means of low-order classical perturbation theory and quantizing it. We used this functional form to determine the parameters of the uniform approximation from trajectory data, and thus improve primitive semiclassical energy eigenvalues. The primitive eigenvalues crossed rather than avoiding each other, whereas the uniform semiclassical eigenvalues show the correct behavior. While the formalism successfully describes the occurrence of avoided crossings and individual eigenvalues, the accuracy of the splittings it yields at the avoided crossings varies due to a central approximation of the treatment, namely using an average value for the Fourier coefficient of the trigonometric term. A close examination of this replacement showed that it gives better results the smaller the fractional differences in quantum numbers. ${ }^{12}$

We also examined the correlation between the width of the classical resonance in $I_{\alpha}$ space and the splittings of the quantum energy levels; and found that whenever the width of the classical resonance was small, the energy level splittings of almost degenerate levels were exponentially small also. These progressively smaller splittings across a sequence of avoided crossings are a classically forbidden phenomenon, and might be termed, as has been done by Davis and Heller, "dynamic tunneling." ${ }^{50}$ It has been suggested to us by Dr. J. N. L. Connor that in the $I_{\alpha}-\alpha$ space in our particular case it corresponds more to a reflection in an "over the barrier" problem, which is, of course, still classically forbidden (cf. Ref. 48). Indeed, this is also largely the case, we find, in the original (Cartesian) $q-p$ space. An analysis of the phase integral solution to Eq. (2.27) will be published elsewhere. In an interesting work on the different problem of local mode splittings, Lawton and Child ${ }^{51}$ connected trajectories of local modes by an approximate tunneling path, which joined the cusps of box-like trajectories. This tunneling proceeded along a real coordinate and with an imaginary momentum.

It should be noted that the avoided crossings dealt with here mix nuclear wave functions, whereas at the betterknown avoided crossings in the collision problem it is the electronic wave functions that interact strongly. An extension of the present treatment to such nonadiabatic collision phenomena might make use of the classical analogs of electronic degrees of freedom. ${ }^{52}$

\section{ACKNOWLEDGMENTS}

We are pleased to acknowledge the support of the present research by the U. S. Department of Energy under contract W-7405-eng-26 with the Union Carbide Corporation (at Oak Ridge) and by a grant from the National Science Foundation (at the California Institute of Technology). We thank Dr. J. N. L. Connor for his helpful comments.

\section{APPENDIX A: DERIVATION OF THE RESONANCE HAMILTONIAN BY BIRKHOFF-GUSTAVSON PERTURBATION THEORY}

Birkhoff, in his treatment of nonseparable classical systems, ${ }^{53}$ gave a procedure to transform the original Hamiltonian into a normal form consisting of a power series of onedimensional uncoupled harmonic oscillator Hamiltonians. This procedure was modified by Gustavson ${ }^{32}$ to include systems with commensurate frequencies. The resulting expressions are particularly easy to quantize ${ }^{25}$ In what follows, we first summarize for the purpose of the present paper, the method along the lines of Gustavson's work. ${ }^{32}$

Consider an $n$-dimensional system with a Hamiltonian

$$
H(u, v)=H^{(2)}(u, v)+H^{(3)}(u, v) \ldots
$$

which is a power series in the coordinates $u=\left\{u_{v}\right\}$ and momenta $v=\left\{v_{v}\right\}$, and $H^{(s)}$ is a homogeneous polynomial of degrees

$$
H^{(s)}(u, v)=\sum_{i+j=s} a_{i j} u^{i} v^{j}, s=2,3, \ldots
$$

In these expressions, $i=\sum_{v=1}^{n} i_{v}$ and $u^{i}$ denotes $\Pi_{v=1}^{n} u_{v}^{i_{v}}$, respectively.

If $H^{(2)}$ is positive definite, it can be canonically transformed to the harmonic oscillator Hamiltonian

$$
H^{(2)}(p, q)=\sum_{k=1}^{n} \frac{\omega_{k}}{2}\left(p_{k}^{2}+q_{k}^{2}\right)
$$

According to Gustavson, ${ }^{32} H(p, q)$ is in normal form if

$$
D H(p, q) \equiv \sum_{k=1}^{n} \omega_{k}\left(p_{k} \frac{\partial}{\partial q_{k}}-q_{k} \frac{\partial}{\partial p_{k}}\right) H(p, q)=0,
$$

i.e., that the Poisson bracket of $H$ with $H^{(2)}$ vanishes. 
The transformation of the power-series Hamiltonian (A1) to normal form can be achieved by successive canonical transformations of the so-called $F_{2}$ type. ${ }^{31}$ To this effect, one defines generating functions

$$
F_{2}^{(s)}(P, q)=\sum_{k=1}^{n} P_{k} q_{k}+W^{(s)}(P, q),
$$

where $\left\{P_{k}\right\}$ are the new momenta and $s \geqslant 3$. The transformation equations are

$$
\begin{aligned}
& Q=q+\frac{\partial W^{(s)}}{\partial P}, \\
& p=P+\frac{\partial W^{(s)}}{\partial q}, \\
& H(p, q)=\Gamma(P, Q)=\sum_{s=2}^{\infty} \Gamma^{(s)}(P, Q),
\end{aligned}
$$

where $Q$ is the new coordinate. $\Gamma^{(2)}(P, Q)$ is $H^{(2)}(p, q)$ evaluated at $p=P, q=Q$. Beginning with $s=3$, the canonical transformations $F_{2}^{(s)}$ given by Eq. (A5) put all terms Eq. (A1) for $H$ of degree $s$ into normal form. $W^{(s)}$ and $\Gamma^{(s)}$ are determined from Eqs. (A12) and (A13) below in the following manner.

The working equations arise from expanding the equality

$$
\Gamma\left(P, q+\frac{\partial W^{(s)}}{\partial P}\right)=H\left(P+\frac{\partial W^{(s)}}{\partial q}, q\right)
$$

in a Taylor series around $(P, q)$ and collecting terms of equal degree $i$. They are

$$
\begin{aligned}
& \Gamma^{(i)}(P, q)=H^{(i)}(P, q), \quad i<s, \\
& D W^{(s)}(P, q)=\Gamma^{(s)}(P, q)-H^{(s)}(P, q), \quad i=s \\
& \Gamma^{(i)}(P, q)=H^{(i)}(P, q)+\sum_{\left\langle j_{v},\right.}\left(\prod_{v=1}^{n} j_{v} !\right)^{-1}\left\{\left[\prod_{v=1}^{n}\left(\frac{\partial W^{(s)}}{\partial q_{v}}\right)^{j_{v}}\right] \frac{\partial^{i} H^{(l)}}{\prod_{v=1}^{n} \partial P_{v}^{j_{v}}}-\left[\prod_{v=1}^{n}\left(\frac{\partial W^{(s)}}{\partial P_{v}}\right)^{j_{v}}\right] \frac{\partial^{j} \Gamma^{(l)}}{\prod_{v=1}^{n} \partial q_{v}^{j}}\right\}, i>s .
\end{aligned}
$$

These terms are terms used to normalize terms in $H$ of degree $s$. In the last equation, the summation is over all combinations $\left\{j_{v}\right\}$, consistent with ${ }^{32}$

$$
1 \leqslant j \leqslant l \text {, }
$$

$$
l \geqslant 2, \quad s \geqslant 3,
$$

$j$ is the sum $\Sigma_{v=1}^{n} j_{v} ; l$ is fixed for a particular combination of the $j_{v}$ 's, and is defined $b^{32}$

$$
l=i-j(s-2) \text {. }
$$

[Incidentally, it follows from Eq. (A9) that $l<i$. The condition $s \geqslant 3$ arises since $H^{(2)}$ is already in normal form. For this reason also, $l \geqslant 2$.] Because of these restrictions, the $\Gamma^{(l)}$ 's in the right-hand side of Eq. (A8c) always have $l<s+1$. Furthermore, $H^{(i)}(P, q)$ and $\Gamma^{(i)}(P, q)$ denote $\left.H^{(i)}(p, q)\right|_{p=P}$ and $\left.\Gamma^{(i)}(P, Q)\right|_{Q=q}$, respectively.

The canonical transformation of degree $s$ leaves all terms $H^{(i)}$ of overall degree $i<s$ [see Eq. (A2)] unchanged, e.g., Eq. (A8a), since they are already in normal form. The normal form is obtained in Eq. (A8b) by choosing $\Gamma^{(s)}$ such that the operator $D^{-1}$ acting on the right-hand side gives a finite result; thus an equation for $W^{(s)}$ is obtained. This generating function, in turn, leads to additional terms of degree $i>s$. The new Hamiltonian, at this stage of the normalization, is denoted by a subscript $s$ and $\Gamma_{s}(P, Q)$.

$$
\Gamma_{s}(P, Q)=\Gamma_{s}^{(2)}+\Gamma_{s}^{(3)}+\Gamma_{s}^{(4)}+\ldots,
$$

where $\Gamma_{s}^{(i)}$ is unaffected by $s$ for all $i<s$. All terms of $\Gamma_{s}$ up to and including degree $s$ are in normal form after the transfor- mation generated by $W^{(s)}$. When using Eqs. (A8) (with $s$ replaced everywhere by $s+1)$ to normalize terms in $\left.\Gamma_{s}(P, Q)\right|_{Q=q}$ of degree $s+1$, each term of the Hamiltonian (A10), $\Gamma_{s}^{(i)}$, must be identified with the $H^{(i)}$ on the right-hand side of Eqs. (A8). [Note, the $\Gamma^{(s)}$ in Eq. (A8b) is now $\Gamma_{s+1}^{(s+1)}$ and the $H^{(s+1)}$ there is equal to $\Gamma_{s}^{(s+1)}$.]

In order to solve for $W^{(s)}$ in $\mathrm{Eq}$. (A8b), one makes a canonical transformation to variables $\left(\eta_{k}, \xi_{k}\right)$ such that ${ }^{32}$

$$
\begin{aligned}
& P_{k}=2^{-1 / 2}\left(\eta_{k}+i \xi_{k}\right), \\
& q_{k}=2^{-1 / 2}\left(\eta_{k}-i \xi_{k}\right) .
\end{aligned}
$$

This changes the operator $D$ into its diagonal form $\tilde{D}$ in $(\xi, \eta)$ space:

$$
\tilde{D}(\xi, \eta)=i \sum_{k} \omega_{k}\left(\xi_{k} \frac{\partial}{\partial \xi_{k}}-\eta_{k} \frac{\partial}{\partial \eta_{k}}\right) .
$$

Since monomials of the form

$$
\Phi_{l m}=\prod_{k} \eta_{k}^{l_{k} \xi_{k}^{m_{k}}}
$$

are eigenfunctions of $\tilde{D}$ with eigenvalues

$$
E_{l m}=i \sum_{k=1}^{n} \omega_{k}\left(m_{k}-l_{k}\right)
$$

they are eigenfunctions of $\tilde{D}^{-1}$ also,

$$
\tilde{D}^{-1} \Phi_{l m}=E_{l m}^{-1} \Phi_{l m} \text {. }
$$

Equation (A8b) can then be solved as

$$
\tilde{W}^{(s)}=\tilde{D}^{-1}\left(\tilde{\Gamma}^{(s)}-\tilde{H}^{(s)}\right) \text {, }
$$


after expressing $\tilde{\Gamma}^{(s)}$ and $\tilde{H}^{(s)}$ in terms of the sum of monomials in $\xi$ and $\eta$ to yield $\tilde{\Gamma}^{(s)}$ and $\tilde{H}^{(s)}$.

To make the right-hand side of Eq. (A12) finite, $\tilde{\Gamma}^{(s)}$ must be chosen such that it will cancel all terms in $\tilde{H}^{(s)}$ which give a zero eigenvalue $E_{l m}$. These are terms for which $m_{k}=l_{k}$ (all $k$ ), regardless of the values of $\omega_{k}$, and, because of the presence of a zero-order resonance, terms such that $E_{l m}$ is proportional to the resonance condition (2.3). Using these two kinds of terms, both referred to as "null space terms," $\tilde{N}^{(s)}$, and the remainder of terms in $\tilde{H}^{(s)}$, referred to as the "range space terms," $\tilde{R}^{(s)}$, we have

$$
\tilde{H}^{(s)}=\tilde{N}^{(s)}+\tilde{R}^{(s)}
$$

We now choose $\tilde{\Gamma}(s)$ so that

$$
\tilde{\Gamma}^{(s)}=\tilde{N}^{(s)} \text {. }
$$

It then follows that

$$
\tilde{W}^{(s)}=-\tilde{D}^{-1} \tilde{R}^{(s)}
$$

Using $\tilde{\Gamma}^{(s)}$ and $\tilde{W}^{(s)}$ and the inverse of the canonical transformation (A11) one obtains $\tilde{\Gamma}^{(s)}$ and $\tilde{W}^{(s)}$, i.e., $\Gamma^{(s)}(q, P)=\tilde{\Gamma}^{(s)}(\xi, \eta)$, etc.

For Hamiltonian (2.8), replacing $p_{k}$ in (2.8) by $\omega_{k}^{0^{1 / 2}} p_{k}$ and $q_{k}$ by $\omega_{k}^{0^{-1 / 2}} q_{k}$, to convert to the notation in Eq. (A3) we obtain

$$
\begin{aligned}
H= & \frac{1}{2} \sum_{k=1}^{2} \omega_{k}^{0}\left(p_{k}^{2}+q_{k}^{2}\right)-a \sum_{k=1}^{2} \omega_{k}^{0^{-3 / 2}} q_{k}^{3} \\
& +\lambda \frac{x^{2} y^{2}}{\omega_{x}^{0} \omega_{y}^{0}}-\frac{b x y^{3}}{\left(\omega_{x}^{0} \omega_{y}^{0^{3}}\right)^{1 / 2}},
\end{aligned}
$$

where $q_{1}$ denotes $x, \omega_{1}^{0}$ denotes $\omega_{x}^{0}$, etc.

Thus, in Eq. (A1) for $H$ we have

$$
\begin{aligned}
& H^{(2)}=\frac{1}{2} \sum_{p=1}^{2} \omega_{k}^{0}\left(p_{k}^{2}+q_{k}^{2}\right), \\
& H^{(3)}=-a \sum_{k=1}^{2} \omega_{k}^{0^{-3 / 2}} q_{k}^{3},
\end{aligned}
$$

and

$$
\begin{aligned}
& H^{(4)}=\lambda \frac{x^{2} y^{2}}{\omega_{x}^{0} \omega_{y}^{0}}-\frac{b x y^{3}}{\left(\omega_{x}^{0} \omega_{y}^{0^{0}}\right)^{1 / 2}} . \\
& H^{(5)}=H^{(6)}=\cdots=0 .
\end{aligned}
$$

The application of Eqs. (A8) to obtain $W^{(3)}$ is as follows: $H^{(3)}$ is converted to $\tilde{H}^{(3)}$. One then separates those terms in $\tilde{H}^{(3)}$ which belong to $\tilde{N}^{(3)}$ from those which belong to $\tilde{R}^{(3)}$, and then use Eq. (A15). This yields

$$
\Gamma^{(3)}=0
$$

and

$$
W^{(3)}=-\frac{a}{3} \sum_{k=1}^{2} \omega_{k}^{0^{-5 / 2}}\left(2 P_{k}^{3}+3 P_{k} q_{k}^{2}\right) .
$$

This $\Gamma^{(3)}$ is now $\Gamma_{3}^{(3)}$. We are next interested in finding $\Gamma_{3}^{(4)}$ : To find this $\Gamma_{3}^{(4)}$, we evaluate Eq. (A8c),

$$
\begin{aligned}
\Gamma_{3}^{(4)}=H^{(4)}(P, q) & +\sum_{k=1}^{2}\left[\left(\frac{\partial W^{(3)}}{\partial q_{k}}\right)\left(\frac{\partial H^{(3)}}{\partial P_{k}}\right)-\left(\frac{\partial W^{(3)}}{\partial P_{k}}\right)\left(\frac{\partial \Gamma^{(3)}}{\partial q_{k}}\right)\right]+\frac{1}{2} \sum_{k=1}^{2}\left[\left(\frac{\partial W^{(3)}}{\partial q_{k}}\right)^{2} \frac{\partial^{2} H^{(2)}}{\partial P_{k}^{2}}-\left(\frac{\partial W^{(3)}}{\partial P_{k}}\right)^{2} \frac{\partial^{2} \Gamma^{(2)}}{\partial q_{k}^{2}}\right] \\
& +\left[\left(\frac{\partial W^{(3)}}{\partial q_{1}}\right)\left(\frac{\partial W^{(3)}}{\partial q_{2}}\right) \frac{\partial^{2} H^{(2)}}{\partial P_{1} \partial P_{2}}-\left(\frac{\partial W^{(3)}}{\partial P_{1}}\right)\left(\frac{\partial W^{(3)}}{\partial P_{2}}\right) \frac{\partial^{2} \Gamma^{(2)}}{\partial q_{1} \partial q_{2}}\right]
\end{aligned}
$$

which simplifies to the following, using Eqs. (A15), (A16) and (A3):

$$
\begin{aligned}
& \Gamma_{3}^{(4)}(P, q)=H^{(4)}(P, q)+\frac{1}{2} \sum_{k=1}^{2}\left[\left(\frac{\partial W^{(3)}}{\partial q_{k}}\right)^{2} \frac{\partial^{2} H^{(2)}}{\partial P_{k}^{2}}-\left(\frac{\partial W^{(3)}}{\partial P_{k}}\right)^{2} \frac{\partial^{2} \Gamma^{(2)}}{\partial q_{k}^{2}}\right], \\
& \Gamma_{3}^{(4)}(P, q)=H^{(4)}(P, q)-\frac{a^{2}}{2} \sum_{k} \omega_{k}^{0^{-4}}\left(q_{k}^{4}+4 p_{k}^{4}\right) .
\end{aligned}
$$

For the present purposes we shall stop this iteration at $s=4$. The procedure described in this appendix can readily be programmed using a symbolic manipulation routine, and we have used the SMP language ${ }^{54}$ to check the present formulas. This program can be used to obtain high-order normal forms of the Hamiltonian (2.8).

The Hamiltonian resulting from the $W^{(3)}$ canonical transformation,

$$
H=H^{(2)}+\Gamma_{3}^{(4)}+\sum_{k=5}^{\infty} \Gamma_{3}^{(k)}
$$

can be put into normal form through terms of fourth degree by applying Eq. (A8) with $s=4$. This normal form is

$$
\begin{aligned}
H= & \sum_{k} \frac{\omega_{k}^{0}}{2}\left(P_{k}^{2}+Q_{k}^{2}\right)-\frac{15 a^{2}}{16} \sum_{k} \omega_{k}^{0-4}\left(P_{k}^{2}+Q_{k}^{2}\right)+\frac{\lambda}{4 \omega_{x}^{0} \omega_{y}^{0}} \\
& \times\left(P_{1}^{2}+Q_{1}^{2}\right)\left(P_{2}^{2}+Q_{2}^{2}\right)-b\left(\omega_{x}^{0} \omega_{y}^{03}\right)^{-1 / 2}\left[Q_{1} Q_{2}\left(Q_{2}^{2}-3 P_{2}^{2}\right)-P_{1} P_{2}\left(P_{2}^{2}-3 Q_{2}^{2}\right)\right] / 8
\end{aligned}
$$


Finally, a transformation into action-angle variables $Q_{k}=\left(2 I_{k}\right)^{1 / 2} \sin \varphi_{k}, \quad P_{k}=\left(2 I_{k}\right)^{1 / 2} \cos \varphi_{k}$ completes the derivation of the resonance Hamiltonian (2.17).

\section{APPENDIX B: AVERAGE QUANTUM NUMBERS FOR USE IN THE RESONANT TERM}

In their recent semiclassical study of intensities of vibrational spectra, ${ }^{55}$ Koszykowski et al. found that for transitions between quantum states $n$ and $m$, semiclassical transition frequencies and intensities agreed well with the corresponding quantum results if they were evaluated at the average action

$$
\bar{I}=\frac{1}{2}\left(I_{n}+I_{m}\right),
$$

corresponding to an "average quantum number" $(n+m) / 2$. This had been noted earlier by Naccache ${ }^{56}$ in the study of the validity of classical mechanics in the derivation of quantummechanical expressions. In particular, this semiclassical correspondence principle leads to exact agreement in the case of Morse oscillators. When studying avoided crossings, we are again dealing with two weakly coupled states, coupled by an internal perturbation rather than by the radiation field, and we use the analogous approximation of replacing $I_{x}$ and $I_{y}$ in the Fourier coefficient of $\cos 2 \alpha$ by their arithmetic averages.

Such a replacement is equivalent to the customary procedure $^{35}$ of evaluating that Fourier coefficient at the center of the nonlinear resonance zone. The "resonance center" for a $m_{x}: m_{y}$ resonance is the pair of actions $\mathbf{I}^{r}=\left(I_{x}^{r}, I_{y}^{r}\right)$ such that

$$
m_{x} \omega_{x}\left(\mathbf{I}^{r}\right)-m_{y} \omega_{y}\left(\mathbf{I}^{\prime}\right)=0 .
$$

When the perturbed angular frequencies $\omega_{i}=\partial H / \partial I_{i}$ are obtained from Eq. (2.27), where the $E$ is written below as $H_{R}$ and where we neglect the $\cos 2 \alpha$ term:

$$
\begin{aligned}
\omega_{x}(\mathbf{I}) & =\partial H_{R} / \partial I_{x} \\
& =\left(\partial H_{R} / \partial I\right)\left(\partial I / \partial I_{x}\right)+\left(\partial H_{R} / \partial I_{\alpha}\right) /\left(\partial I_{\alpha} / \partial I_{x}\right) \\
\omega_{y}(\mathbf{I}) & =\partial H_{R} / \partial I_{y} \\
& =\left(\partial H_{R} / \partial I\right)\left(\partial I / \partial I_{y}\right)+\left(\partial H_{R} / \partial I_{\alpha}\right)\left(\partial I_{\alpha} / \partial I_{y}\right) .
\end{aligned}
$$

The actions at the resonance center satisfy

$$
3 \omega_{y}\left(\mathbf{I}^{r}\right)-\omega_{x}\left(\mathbf{I}^{\prime}\right)=0 .
$$

The substitution of Eqs. (B3) into Eq. (B4), and noting that $I$ is given by Eq. (2.12), gives

$$
\left(\partial H_{R} / \partial I_{\alpha}\right)\left(3 \partial I_{\alpha} / \partial I_{y}-\partial I_{\alpha} / \partial I_{x}\right)=0 .
$$

Using Eq. (2.12), one sees that $\partial H_{R} / \partial I_{\alpha}$ vanishes at the resonance center. Thereby, from Eq. (2.27) we have at the resonant center

$$
\beta=I_{\alpha}^{r}=2 I_{x}^{r},
$$

where the second equality arises from Eq. (2.12). From Eq. $(4.25)$ we have

$$
\beta=n_{x}^{(1)}+n_{x}^{(2)}+1=I_{x}^{(1)}+I_{x}^{(2)} .
$$

Using the definition of the average action $\bar{I}_{x}$, [Eq. (B1)], it is seen that

$$
\beta=2 \bar{I}_{x},
$$

thereby showing the equality

$$
I_{x}^{r}=\bar{I}_{x}
$$

and hence that $I_{y}^{r}=\bar{I}_{y}$.

The replacement of the Fourier coefficient by its value at the resonance center, like other averaging procedures, is expected to be valid whenever variations in $\left|I_{i}-I_{i}\right|$ when $\alpha$ varies are small. For transitions in which the quantum numbers vary extensively, however, it will lead to substantial errors. We illustrate this point in Table $\mathrm{I}$.

\section{APPENDIX C: CROSSING OF PRIMITIVE SEMICLASSICAL EIGENVALUES}

In this appendix we show that the two primitive semiclassical energy eigenvalues, contained in the integral (3.12), cross in the eigenvalue vs $\lambda$ plots. Integral (3.12) gives

$$
\beta \pi \pm T_{1,2}=2 \pi\left(n_{x}^{(1,2)}+\frac{1}{2}\right)
$$

where

$$
T_{1,2}=\int_{0}^{\pi}\left[C+\beta^{2}-D E^{(1,2)}-2 q \cos 2 \alpha\right]^{1 / 2} d \alpha .
$$

Thus the two distinct equations for the energies are given, in implicit form, as

$$
\begin{aligned}
& T_{1}=2 \pi\left(n_{x}^{(1)}+\frac{1}{2}\right)-\beta \pi, \\
& -T_{2}=2 \pi\left(n_{x}^{(2)}+\frac{1}{2}\right)-\beta \pi .
\end{aligned}
$$

However, at the special value of

$$
\beta=n_{x}^{(1)}+n_{x}^{(2)}+1
$$

one finds from Eq. (C3) that $T_{1}$ equals $T_{2}$. Since $T$ is a monotonic function of $E$, [cf. Eqs. (3.12), (2.29), and (3.30)] one concludes that when $T_{1}=T_{2}$ then $E^{(1)}$ and $E^{(2)}$ are equal, i.e., the primitive semiclassical energies cross.

a) Present address: Department of Chemistry, University of Colorado, Boulder, Colorado 80309

${ }^{\circ}$ Contribution No. 6837.

'For example, M. S. Child, in Atom-Molecule Collision Theory, edited by R. B. Bernstein (Plenum, New York, 1979), p. 427.

${ }^{2}$ T. G. Heil, S. E. Butler, and A. Dalgarno, Phys. Rev. A 27, 2365 (1983).

${ }^{3}$ S. Bienstock and A. Dalgarno, J. Chem. Phys. 78, 224 (1983).

${ }^{4}$ M. S. Child, in Molecular Spectroscopy, edited by R. F. Barrow, D. A. Long, and D. J. Miller (The Chemical Society, London, 1974), Spec. Period. Rep. 2, 466

${ }^{5}$ R. A. Marcus, in Horizons in Quantum Chemistry, Proceedings of the 3rd International Congress on Quantum Chemistry, Kyoto, 1979, edited by K. Fukui and B. Pullman (Reidel, Dordrecht, Netherlands, 1980), p. 107. ${ }^{6} D$. W. Noid, M. L. Koszykowski, M. Tabor, and R. A. Marcus, J. Chem. Phys. 72, 6168 (1980), cf. D. W. Noid, M. L. Koszykowski, and R. A. Marcus, Chem. Phys. Lett. 73, 269 (1980).

${ }^{7}$ R. A. Marcus, Ann. N.Y. Acad. Sci. 357, 169 (1980).

${ }^{8}$ R. Ramaswamy and R. A. Marcus, J. Chem. Phys. 74, 1379 (1981).

${ }^{9}$ M. V. Berry, Ann. Phys. N. Y. 131, 163 (1981).

${ }^{10}$ P. J. Richens and M. V. Berry, Physica 2D, 495 (1981). These authors studied overlapping avoided crossings for a new type of system termed "pseudo-integrable."

"D. W. Noid, M. L. Koszykowski, and R. A. Marcus, Annu. Rev. Phys. Chem. 32, 267 (1981).

${ }^{12}$ D. W. Noid, M. L. Koszykowski, and R. A. Marcus, J. Chem. Phys. 78, 4018 (1983).

${ }^{13}$ R. A. Marcus, Faraday Discuss. Chem. Soc. London (in press, 1983).

${ }^{14} \mathrm{R}$. A. Marcus, in Picosecond Phenomena III, edited by K. B. Eisenthal, R. 
M. Hochstrasser, W. Kaiser and A. Laubereau (Springer, New York, 1982), p. 154.

${ }^{15}$ B. V. Chirikov, Phys. Rep. 52, 263 (1979).

${ }^{16}$ D. Farrelly and W. P. Reinhardt, J. Chem. Phys. 78, 606 (1983).

${ }^{17}$ J. Ford, Adv. Chem. Phys. 24, 155 (1973); J. Ford, in Fundamental Problems in Statistical Mechanics, edited by E. G. D. Cohen (North-Holland, Amsterdam, 1975), Vol. 3, p. 215.

"I. C. Percival, Adv. Chem. Phys. 36, 1 (1977).

${ }^{19}$ M. V. Berry, Am. Inst. Phys. Conf. Proc. 46, 16 (1978).

${ }^{20}$ M. Tabor, Adv. Chem. Phys. 46, 73 (1981).

${ }^{2}$ 'S. A. Rice, Adv. Chem. Phys. 47, 117 (1981).

${ }^{22}$ P. Brumer, Adv. Chem. Phys. 47, 201 (1981)

${ }^{23}$ W. Eastes and R. A. Marcus, J. Chem. Phys. 61, 4201 (1974); D. W. Noid and R. A. Marcus, ibid 62, 2119 (1975); K. S. Sorbie, Mol. Phys. 32, 1577 (1976); K. S. Sorbie and N. C. Handy, ibid. 32, 1327 (1976); D. W. Noid and R. A. Marcus, J. Chem. Phys. 62, 2119 (1977); K. S. Sorbie and N. C. Handy, Mol. Phys. 33, 1319 (1977); D. W. Noid, M. L. Koszykowski and R. A. Marcus, J. Chem. Phys. 71, 2864 (1979); 73, 391 (1980).

${ }^{24}$ S. Chapman, B. C. Garrett, and W. H. Miller, J. Chem. Phys. 64, 502 (1976); I. C. Percival and N. Pomphrey, Mol. Phys. 31, 97 (1976); J. Phys. B 9, 3131 (1976); Mol. Phys. 35, 649 (1978); G. C. Schatz and M. D. Moser, Chem. Phys. 35, 239 (1978); G. C. Schatz and T. Mulloney, J. Phys. Chem. 83, 989 (1979); C. Jaffé and W. P. Reinhardt, J. Chem. Phys. 71, 1862 (1979).

${ }^{25}$ R. T. Swimm and J. B. Delos, J. Chem. Phys. 71, 1706 (1979).

${ }^{26}$ The divergence of the series is related to the nonintegrability of the Hamiltonian; see C. Siegel, Ann. Math. 42, 806 (1941). An example occurs in G. Contopoulos, L. Galgani, and A. Giorgilli, Phys. Rev. A 18, 1183 (1978).

${ }^{27} \mathrm{M}$. Born, Mechanics of the Atom (Ungar, New York, 1960).

${ }^{28} \mathrm{C}$. Siegel and J. Moser, Lectures on Celestial Mechanics (Springer, New York, 1971); C. R. Eminhizer, R. H. G. Hellemann, and E. W. Montroll, J. Math. Phys. 17, 121 (1976).

${ }^{29}$ G. M. Zaslavskii and B. V. Chirikov, Sov. Phys. Usp. 14, 549 (1972); E. V. Shuryak, Sov. Phys. JETP 44, 1070 (1976).

${ }^{30}$ Y. Weissmann and J. Jortner, J. Chem. Phys. 77, 1469 (1982).

${ }^{31}$ H. Goldstein, Classical Mechanics (Addison-Wesley, Reading, Mass., 1950).

${ }^{32}$ F. G. Gustavson, Astron. J. 71, $670(1966)$.

${ }^{33} \mathrm{~F}$. Gantmacher, Lectures in Analytical Mechanics (Mir, Moscow, 1979), p. 130.

${ }^{34}$ The last equality in Eq. (2.21) arises in the following way. Since $I_{\alpha}=2 I_{x}$ and $2 \alpha=\varphi_{x}-3 \varphi_{y}+\pi$, for $\varphi_{y}=$ constant (usually 0 ),

$\int_{0}^{\pi} I_{x} d \alpha=\int_{-\pi / 2}^{\pi / 2} 2 I_{x} d\left(\frac{\varphi_{x}}{2}\right)=\int_{-\pi}^{\pi} I_{x} d \varphi_{x}$

${ }^{35}$ E. L. Sibert III, W. P. Reinhardt, and J. T. Hynes, J. Chem. Phys. 77, 3583 (1982).

${ }^{36}$ P. F. Byrd and M. D. Friedman, Handbook of Elliptic Integrals, 2nd ed. (Springer, New York, 1971); I. S. Gradshteyn and I. W. Ryzhik, Table of
Integrals Series and Products (Academic, New York, 1965).

${ }^{37}$ D. W. Noid and R. A. Marcus, J. Chem. Phys. 67, 559 (1977).

${ }^{38}$ If $\alpha$ does not go through it full range of $\pi$, as in the cases labeled $b$ in Table III, no value is reported there for the primitive semiclassical approximation.

${ }^{39}$ To actually determine $S$, in practice, we find a trajectory which in $p_{x}-x$ space is just larger than the outside branch of the separatrix, and one which is just smaller than the inside branch. The difference of these two cases yields $S$, to an accuracy of about $1 / 500$, an accuracy less than that for determining the phase integrals themselves.

${ }^{40}$ In order to check the consistency of possibly neglecting the trigonometric term, we first evaluated $C$ and $D$ from the perturbation expressions (3.7) and (3.8). Then, using these, we performed a primitive semiclassical quantization (explained in Sec. V B) with $b=0.005$ and $b=0.02$, an appreciable perturbation. This procedure takes full account of the trigonometric term, and in that sense is the perturbation equivalent of the classical trajectory quantization. We then use these primitive semiclassical energies to obtain a new $C$ and $D$. As noted in the text, the differences between such $C$ 's and $D$ 's and the original ones were minor.

${ }^{41}$ For example, R. A. Marcus, Chem. Phys. Lett. 7, 525 (1970); J. Chem. Phys. 54, 3965 (1971).

${ }^{42}$ N. W. McLachlan, Theory and Applications of Mathieu Functions (Clarendon, Oxford, 1947).

${ }^{43}$ Handhook of Mathematical Functions, edited by M. Abramowitz and I. A. Stegun (Dover, New York, 1965).

${ }^{44}$ H. Eyring, J. Walter, and G. E. Kimball, Quantum Chemistry (Wiley, New York, 1944).

${ }^{45}$ T. Tamir, Math. Comp. 16, 100 (1962).

${ }^{46}$ Developed by National Activity to Test Hardware, cf. Lecture Notes in Computer Science, edited by G. Goos and J. Hartmanis (Springer, Berlin, 1976), Vol. 6.

${ }^{47}$ L. F. Shampine and M. K. Gordon, Computer Solution of Ordinary Differential Equations, The Initial Value Program (Freeman, San Francisco, 1975).

${ }^{48}$ E. L. Sibert III, J. T. Hynes, and W. P. Reinhardt, J. Chem. Phys. 77, 3595 (1982).

${ }^{49} \mathrm{C}$. Jaffé and W. P. Reinhardt, J. Chem. Phys. 77, 5191 (1982)

${ }^{50}$ M. J. Davis and E. J. Heller, J. Chem. Phys. 75, 2461 (1980).

${ }^{51}$ R. T. Lawton and M. S. Child, Mol. Phys. 44, 709 (1981).

${ }^{52}$ H. D. Meyer and W. H. Miller, J. Chem. Phys. 70, 3214 (1979).

${ }^{53}$ G. D. Birkhoff, Dynamical Systems (Am. Math. Soc., New York, 1966), Vol. IX.

${ }^{54} \mathrm{SMP}$ is a symbolic manipulation program developed by the High Energy Physics group at Caltech. The SMP Handbook, published as an internal report in 1981, and further information can be obtained from SMP, Mail Code 356-48, California Institute of Technology, Pasadena, CA 91125.

${ }^{55}$ M. L. Koszykowski, D. W. Noid, and R. A. Marcus, J. Phys. Chem. 86, 2113 (1982).

${ }^{56}$ P. F. Naccache, J. Phys. B 5, 1308 (1972). 